\title{
MAKER: Redesign of a Multipurpose Hardware Tool to Improve its Func- tionality and Marketability
}

\section{Mr. David Alexander IV, Tuskegee University \\ Dr. Vimal Kumar Viswanathan, Tuskegee University}

Dr. Vimal Viswanathan is an assistant professor in the Mechanical Engineering Department at Tuskegee University. He earned his Ph.D. from Texas A\&M University. His research interests include design innovation, creativity, design theory and engineering education.

Ms. Shraddha Sangelkar, Pennsylvania State University, Erie

Shraddha Sangelkar is an Assistant Professor of Mechanical Engineering at Penn State Erie, The Behrend College. She is the senior design co-ordinator for Mechanical Engineering within the School of Engineering at Penn State Behrend. She is also involved in the executive committee of Design In Engineering Education Division at ASEE and the Capstone Design Conference.

Johnathan Moody, Pennsylvania State College, Erie 


\title{
MAKER: Redesign of a Multipurpose Hardware Tool to Improve its Functionality and Marketability
}

\begin{abstract}
$\underline{\text { Abstract }}$
This paper presents the summary of a maker project that redesigned a multi-purpose utility tool, originally designed for linemen. While the tool had a great potential, its original design was a letdown and the product was a failure in the market. Applying a systematic procedure, the authors redesigned said tool. During the analysis of the original design, it was observed that the viewpoints of the customers (linemen) were not considered for the design of the tool. The major disadvantages included the heavy weight and the improper weight distribution of the tool. The tool also contained a feature that was not of much use for the linemen. In addition, the biomechanics aspects were not considered for the design. In the redesign cycle, the customers are also included and they provided very valuable insights for the redesign. This paper presents all the steps involved along with the details of the final redesigned tool.
\end{abstract}

\section{Introduction}

The primary objective of this maker project was to redesign a multi-purpose tool that is intended for both everyday and professional purposes. The existing product is a multipurpose utility tool titled the "Goat Wrench" (Shown in Figure 1). This multipurpose tool is available in stores and online for everyday consumers and distributors. The "Goat Wrench" is 12" long and weighs 1.65 lbs. It has a built-in hammer, bolt threaders, and multi-size grip jaws with self- ratchet action. This multipurpose product is designed to provide its users better storage and utilize three tool functions more conveniently with the goal to optimize time and conserve energy. The goat wrench is popular among linemen who work on utility poles for power companies. It allows them to complete their pole maintenance tasks more effectively and efficiently without the hassle of transporting and storing multiple tools. Especially when having to physically scale the power poles, which is in the job description of a utility lineman, a multipurpose product is ideal. This allows them to carry a single tool with them that replaces multiple tools.

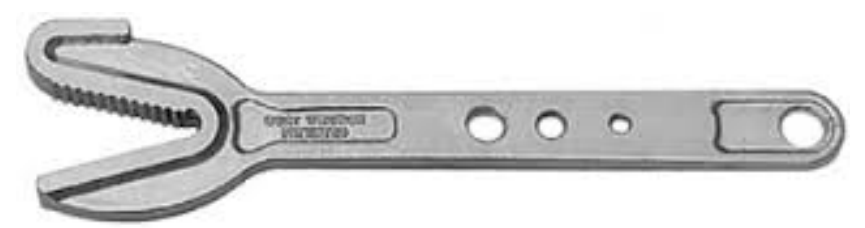

Figure 1. Goat Wrench Multifunction Tool

The goat wrench is a great concept and there is a great potential in this product, but its design contains several flaws. Due to the first author's prior experience in the power utility space, some misconceptions in the product were identified at the first glance. At a utility company, it is common practice to discard all the current equipment on a utility pole and replace them with new equipment. With that knowledge, the bolt threaders were viewed as a useless function for the prominent end user. The functions of the tool appeared to have been selected arbitrarily without the consideration of its customers and end users. According to the feedback from the users, the tool was too heavy and too expensive. The sales of the product was not as expected by the 
manufacturer. This was the main reason for the manufacturer to approach the Pennsylvania State University, Erie Campus and Tuskegee University for the redesign of said tool. Authors Alexander and Moody, both undergraduate students, collaboratively handled the project. The students took complete responsibility of the project with occasional updates to their faculty supervisors. We completed all the steps in the design project through weekly meetings and sharing of the materials online. Please note that, although we use some of the design techniques we learned in classrooms, this project was completely controlled by undergraduate students and was not a part of any formal coursework. This is the main reason said project was considered as a maker project.

From the initial analysis of the tool, it appeared that no ergonomic factors were considered in the original design to assure optimum performance and safety of the users. The angles of the teeth were randomly selected although the jaws with the self-ratchet function did work. With an engineering analysis of the "teeth" inside the jaws, optimum performance could be achieved. The customer segment for the tool was too broad. We figured that with a well-defined targetcustomer population, this redesigned tool can re-enter the market and become dominant in that space. It can then expand to other market segments once it has gained enough customers and momentum. This is a simple strategy of divide and rule (conquer), which is used in politics, sociology, and economics.

A redesign and re-launch of the goat wrench was a great idea because there was so much potential in this multipurpose product and with the right design process, it could be a highly preferred product in the market. The selection of individual tools on the goat wrench were commonly used by certain consumers, but there were more unknowns that needed to be analyzed, evaluated, and validated to take the full advantage of the potential of this tool. The customer segment needed to be refined and the primary functions needed to be analyzed and evaluated. These were especially important since there was speculation of the lack thereof. For a design to be successful in the market, the end user's desires and needs must be integrated as much as possible ${ }^{1}$ without overpopulating the design and destroying its unique value.

\section{The Re-design Procedure}

\section{Problem Clarification}

In the design process, we initially defined the problem with the goat wrench as, too poorly designed to be successful in the market. For the problem clarification step, we listed issues utilizing the technical specifications including the tool feedback provided by the sponsor. The goat wrench is 12" long, $1.65 \mathrm{lbs}$., made of forged 8620 steel, with a built in hammer, bolt threaders, and multi-size grip jaws with self- ratchet action. After analyzing the tool and its technical specifications, we understood that the goat wrench consisted of the useless bolt threading function. The functions appeared to be selected arbitrarily without proper reasons. According to feedback provided by the manufacturer, the tool was too expensive and heavy. The manufacturer also informed us that in the original design no ergonomics were considered to achieve optimum performance and safety, no engineering analysis was conducted on the ratchet teeth to achieve optimum performance, no market analysis was conducted, nor a proper customer segmentation. Overall, the goat wrench was designed with minimal analysis, evaluation and 
user/customer consideration, and then manufactured with hopes of being a successful multipurpose product.

\section{Benchmarking}

Next, we used the benchmarking techniques to compare the individual functions with some current products that satisfy said functions. The individual functions of the goat wrench are displayed in Table 1. This table also shows the corresponding individual products. This step allowed us to understand the pros and cons from each individual tool, so that the pros could be integrated accordingly. One main complaint about the product was about the weight of the product. We understood that these problems could be addressed simply by changing the material to an aluminum alloy to reduce the weight. Aluminum alloy would be more expensive but the cost of the product was not a major concern for the manufacturer if it was well designed.

The benchmarking process revealed some additional problems that were not considered initially: the lack of shock absorption, the handle could easily to slip out of hand, and the poor weight distribution within the body of the tool. By incorporating a thermoplastic grip for shock absorption and handle traction, we could make the tool more ergonomic. Similarly, by removing unused bolt threading function, we could redistribute the weight of the tool. Doing so would make room for the addition of a retracting mechanism or a function to secure the goat wrench to an attachment. Also the tool could have a detachable head to assemble corresponding functions for specific pole tasks. We identified these solutions from an analytical perspective. These solutions targeted to provide convenience and comfort to the end users for whatever purpose they use the tool for. The end user suggestions would be compared and contrasted against the engineering designer opinions to finalize what function were most important and feasible. This procedure was performed to ensure the maximum efficiency of the design process.

Table 1. Benchmarking of individual functions of the Goat Wrench against the single-purpose tools used for the same functions ${ }^{2-4}$

\begin{tabular}{|l|l|}
\hline Hammer & \\
\hline Adjustable Wrench & \\
\hline Thread Restorer & \\
\hline
\end{tabular}




\section{Pre-Idea Generation}

The next step in the design procedure was idea generation. This was where random concept variations were sketched to cover a range of possibilities. We used the traditional brainstorming technique $^{5}$ to generate our ideas. It was important to make each concept variation as different as possible from one another. These concepts must also be feasible. This step was to bring the abstract, but creative presence into the design, which was very important in a redesign task. Having the different variations made it easier to visualize what aspects of the design were better than others. It was important to get user feedback on these concepts as well.

After breaking down the goat wrench into its individual functions, we were able to take the highlights from each tool and focus on how the design manipulations could be accomplished without compromising the value of successful tool functions. It was extremely important to produce some feasibly creative "out of the box" concepts in preparation for the end user interviews with the linemen at the utility company. The idea generation was conducted before and after the interview with the linemen. The pre-idea generation allowed us to develop multiple concepts to be critiqued by the experienced linemen. It also set the tempo and direction of effectively and innovatively redesigning the goat wrench.

\section{User Interviews}

The next step in the redesign procedure was the customer feedback on the ideas. We took the ideas generated during the pre-idea generation session to the linemen at a local utility company. This was done to incorporate the feedback, thoughts, and feelings of the end users in the redesign process. This provided further insights useful for the design process. It also helped us to develop early adopters of the concept, which increases the chances of market domination.

The customer interviews were done in two stages. During the first stage, we asked the customers about their experiences with various tools that they used in their day-to-day jobs. The idea was to understand what they liked and disliked about each tool. This was followed by a discussion on the original goat wrench tool and the ideas generated for its redesign. This got their minds churning on the concepts shown and allowed them to think more openly and subjectively about how the product could be improved. They could also provide logic on why or why not certain aspects of the designs were acceptable.

The linemen, who were the prominent users for the goat wrench, gave many insights from an experienced user's perspective. They mentioned specific functions and additions they would like to see on the redesigned goat wrench that would make their everyday jobs convenient. The linemen also introduced a game-changing challenge during the interviews. They mentioned that it was difficult to see the tools when working under machines in the dark. If we could incorporate a mechanism to solve this issue, the product would be extremely useful for them.

\section{Post-Idea Generation}

Taking advantage of all the information expressed during the interview, the post idea generation session was performed with more insights to what really matters to the end user when performing their daily tasks. Similar to the pre-idea generation session, we sketched random idea 
variations for the goat wrench. These new sketches were significantly different from those generated during pre-idea generation as we had a better awareness about the customer preferences. Also awareness has been brought to situations and conditions on how frequently different uses of the tool occurred. Further, the discussion about special situations gave us a deeper understanding about the emergency functionalities required in the product. In summary, at this point, the designers knew what the users wanted in the product, and now they had to include as much functionality as possible.

\section{Final Design}

Lastly, following the post-idea generation, there was a down-selection process to decide which of the concept variations was the best to satisfy the users' wants and needs. With the help of the entire group, including the faculty, we discussed about each concept to arrive at a final design. The final concept was developed with many new exciting features. The material of the final design is made of aluminum 7068 alloy for being lightweight. We decided to use an aluminum alloy instead of the steel material that was previously used. The original weight of the Goat Wrench was approximately $2 \mathrm{lbs}$. using the density of steel $\left(7850 \mathrm{~kg} / \mathrm{m}^{3}\right)$ and the density of aluminum $7068\left(2800 \mathrm{~kg} / \mathrm{m}^{3}\right)$ we can expect that the weight will decrease by $64 \%$ to approximately $0.7 \mathrm{lbs}$. When selecting an aluminum alloy, it is important to not compromise the integrity of the structure. This tool will be subjected to impact loading due to the hammer function. It is important that the material does not fail during the hammer action. Currently, the tool is being optimized from bio-mechanics perspective to reduce shock on the hand. On the same lines the details finite Element analysis for impact loading is work in progress. The material change is an immediate solution to satisfy the issues of high weight given by the manufacturer, but it is wise to exceed expectations. The feedback received from the user interviews is used to add new features to the device. The new features entail, a staple remover, an all in one screwdriver, a hammer, a multi-size grip jaws with self- ratchet action, an integrated ruler, the glow in the dark capabilities, and contoured grips for all the different functions. Figure 2 shows the CAD model of the final design selected. 


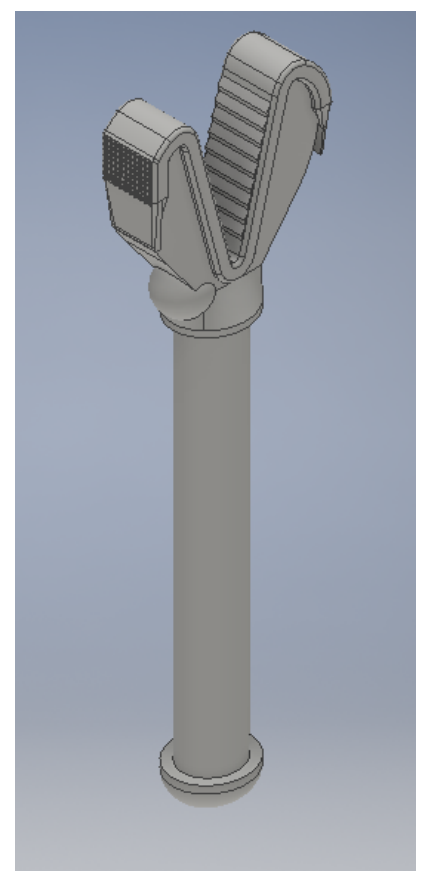

Figure 2. The CAD model of the final design selected for the modified goat wrench

The linemen have to remove staples very frequently when they work on electric poles. This is the primary reason for the inclusion of the staple remover function in the final design. The staple remover is shown in Figure 3. Currently they use a knife to pop open the staples, but this is not the primary function of the blade. Using the blade as a staple remover diminishes the quality of the blade as it is continually used for its secondary function. The blade sharpness deteriorates and it becomes bent and blunt.

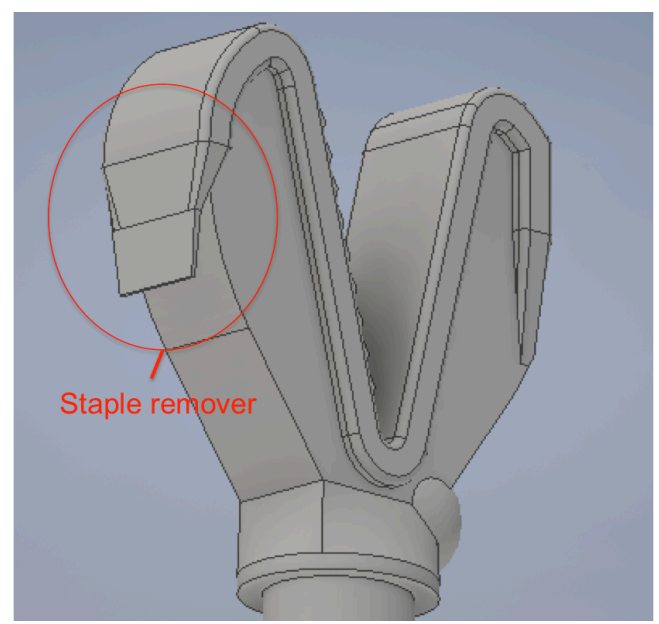

Figure 3. The staple remover feature of the new design

Another tool that is necessary for the linemen to complete their tasks is an all-in-one screwdriver. This is added as another new function in the goat wrench. Due to the compactness of the screwdriver function, it was integrated to occupy a vacancy of leftover space on new design. In addition, the screwdriver function will reduce additional tools that would be needed at some point during the pole maintenance. 
The hammer function is retained in the final design because it is noted as a necessary tool for linemen to complete their tasks on the pole. This was a primary function on the original design. The final design has some slight modifications made to improve its effectiveness, as shown in Figure 4. Instead of a smooth surface, we integrated a rough waffle interface. It is designed to reduce slip when using the hammer on the pole. It serves not only as a way to make its use more effective, but it also provides increased safety. Additionally, due to the material change, the hammer will be lighter and easier allowing the users to conserve energy when performing tasks on the utility pole.

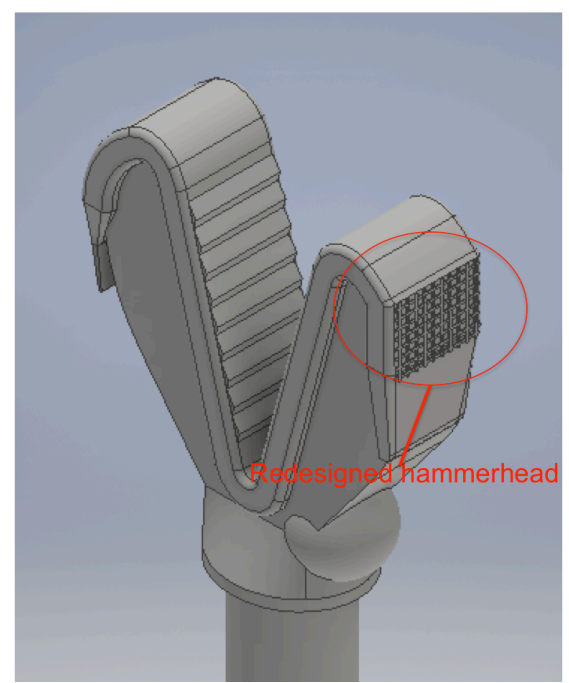

Figure 4. The modified hammer head in the new design

In the original design of the goat wrench, it was observed that the teeth acted as a unidirectional wrench that functions like a ratchet. The V-shaped grip could work on multiple sizes of hexagonal or square nuts. This unidirectional feature is included in the final design as shown in Figure 5. The original design worked better for hexagonal nuts and performed, although not optimally, for square nuts. There is no literature available to support the unidirectional multi-size grip design. The teeth geometry is currently being reverse-engineered to be optimum for square nuts, which are most common on the utility pole operations. The teeth geometry design is workin-progress and it being geometrically optimized to hold on to multiple sizes of square nuts in one direction and slip out in the other direction. It serves as a more effective channel-lock, which is a plier with adjustable locking jaws. With the multi-size grip jaws, the engineered teeth design grips a variety of bolt sizes. This allows the users to tighten or loosen bolts effectively and efficiently. 


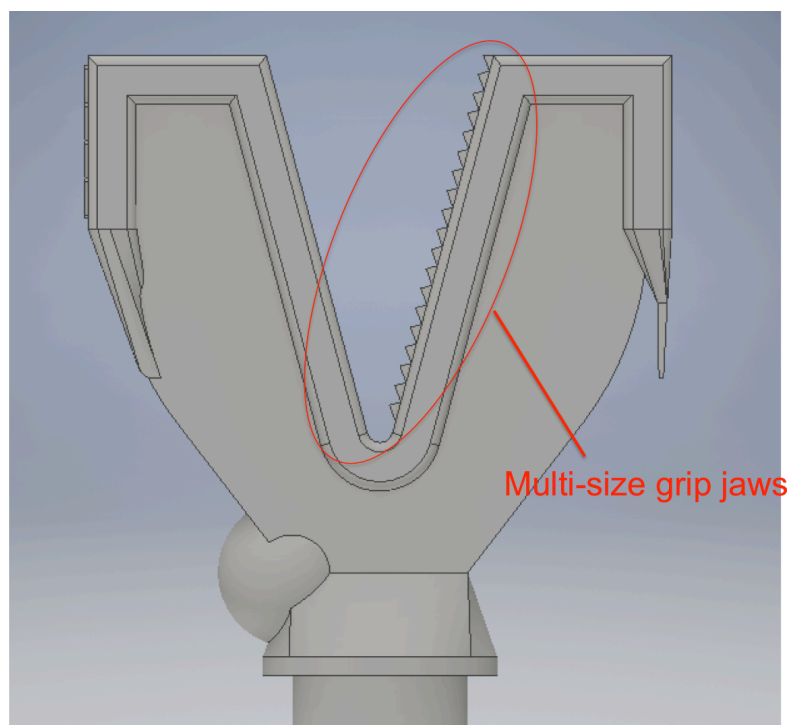

Figure 5. The multi-size grip jaws of the new design

The integrated ruler is another important function added to the final design (not shown in the CAD model). When adding new equipment to the utility poles, the linemen have specifications for the placement of where and how every part gets added to the pole. Typically, linemen use tool dimensions to estimate the distance they need between given parts. These measurements need not be very precise they just need rough estimates. Now, with the integrated ruler they will be able to quickly measure the distance. This function adds effectiveness to the work of the linemen. Figure 6 shows the conceptual sketch of the grip and with the scale markings.

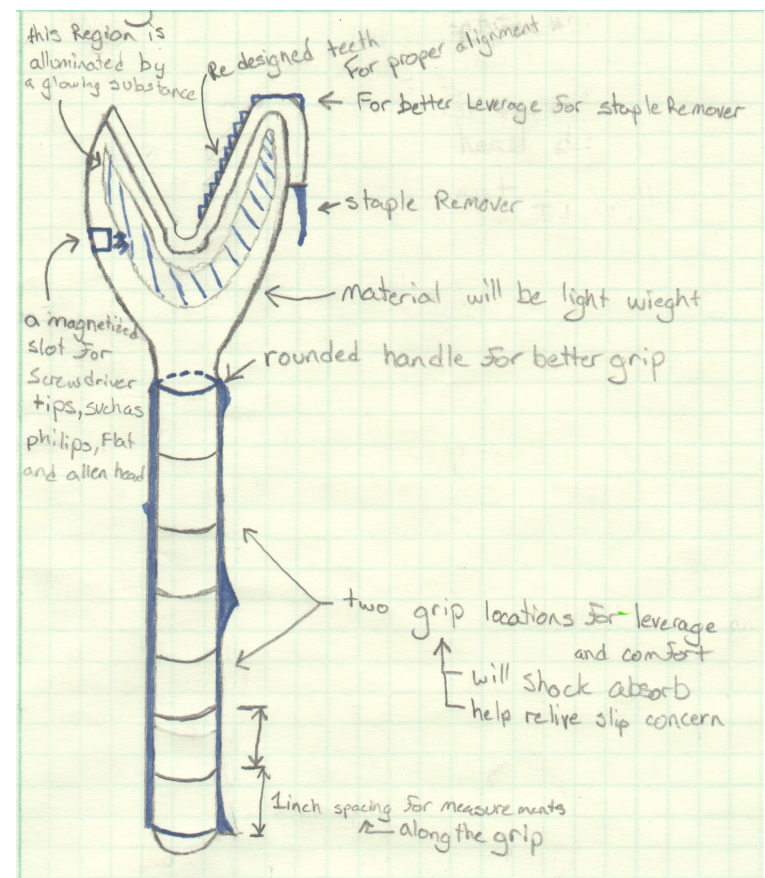

Figure 6. Conceptual sketch of the grip and scale

The final design also glows in the dark. It is common that linemen work on utility poles during storms and sometimes there are power outages which result in them fixing equipment in 
complete darkness. Even when there are lights provided to help the linemen, there are some cases where it is still dark under the equipment and they cannot see the tool they are working with. With that information, it would be a great function to have on standby for such an unexpected situation. The glow in the dark property for the goat wrench is applied using a powder coat process that will cover the wrench as a thin durable skin. The phosphorescent material is simply charged by light and when placed in darkness it glows. It is a process where energy the absorbed by a substance is released slowly in the form of light. It can last up to a few hours in a dark room after charged by light. Game changing functions like this allows the multipurpose product to serve its purpose by being convenient in a wider range of situations including the unexpected ones and allowing users to perform effectively.

The versatile contoured grips are selected as a final function to provide shock absorption, traction, and comfort when using each function (Figure 7). The grips will be modeled on the 3-D printed model with modeling clay after printing to demonstrate the grip and scale feature. The grip is oval in cross-section compared to the original rectangular cross-section. The shock absorption is very important when it comes to safety and effectiveness. The tasks of the linemen are repetitive, which increases the risk of developing nagging long lasting injuries. This hinders the effective work life of the linemen. Tools that are taxing to the body leaves them with medical issues to deal with when retire from the job. Hence, the shock absorption feature from biomechanics perspective is currently is being worked on. Traction that the grip provides is also important because slip is a common issue with the tools, which could result in a serious injury. This problem is amplified since majority of the tools are made of steel. It also adds unnecessary inconveniencing difficulty to the job when the tools are constantly slipping when the linemen are trying to work. The versatility of the grip is a huge factor. It provides the necessary comfort when using the different functions. The increased diameter is ergonomic for the screwdriver function, the hand slots provide stability and comfort when using the hammer, wrench, and staple remover. The grip adds efficiency and effectiveness to the tool.

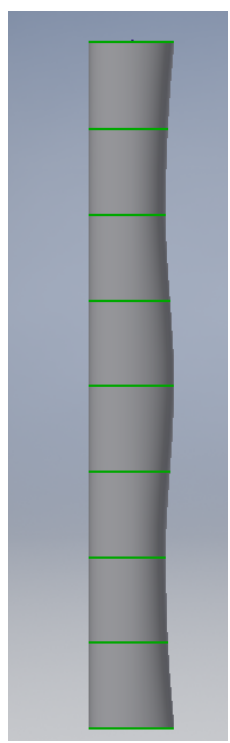

Figure 7. The redesigned contoured grips for the new goatwrench 


\section{Summary \& Lessons Learned}

Customer's today make product decisions based on subjective factors: convenience, ease of use, and cost. They spend their money on what "feels" right and are often unable to provide an explanation why. If the customer's feelings are considered while designing a product, its market success can be ensured. Customers buy what they want, whether it is the best financial decision or not.

We learned that it essential to include the customer in the design process. The original goat wrench was a product in which the customer requirements were not considered. During the redesign, we approached the intended end users and they provided valuable insights that might lead to the market success of the product.

\section{The Path Forward}

Currently, we are in the process of creating a non-functional prototype of the final design with 3D printing technology. Biomechanics analysis for shock absorption and the optimum teeth geometry design is currently work-in-progress. This will be used to convey the final design to the sponsor and device manufacturing strategies. During the demonstration of this paper, the nonfunctional 3-D printed prototype will be presented along with the original design for comparison.

\section{Acknowledgements}

For this project, the Undergraduate Student Research Grant of Penn State Behrend has provided student support. In addition, we would like to acknowledge Ron Anderson from Anderal Technologies for providing industry support and feedback for the redesign of goat wrench. We would also like to acknowledge the Alabama Power Company for letting us interview their linemen as a part of our data collection.

\section{References:}

1. Otto, K., \& Wood, K. (2001). Product design: techniques in reverse engineering and new product design. Prentice-Hall.

2. Claw Hammer, available at http://deadliestfiction.wikia.com/wiki/Claw_Hammer; accessed on 01/31/2016

3. Tools Delivered, available at http://www.toolsdelivered.com/Williams-13406A-Hand-Tools-WrenchesAdjustable-Standard-Grip; accessed on 01/31/2016

4. Smartshopbuy, available at http://smartshopbuy.com/Tools/textile-sack-coarse-mesh-25-kg-50-x-80-x-100.htm; accessed on 01/31/2016

5. Rawlinson, J. G. (1981). Creative thinking and brainstorming. Farnborough, Hants: Gower. 